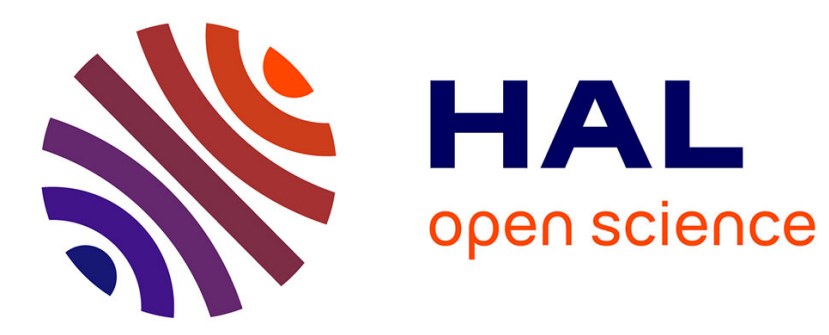

\title{
Online conferences - Towards a new (virtual) reality
}

Goedele Roos, Julianna Oláh, Rebecca Ingle, Rika Kobayashi, Milica Feldt

\section{To cite this version:}

Goedele Roos, Julianna Oláh, Rebecca Ingle, Rika Kobayashi, Milica Feldt. Online conferences Towards a new (virtual) reality. Computational and Theoretical Chemistry, 2020, 1189, pp.112975. 10.1016/j.comptc.2020.112975 . hal-03044982

\section{HAL Id: hal-03044982 \\ https://hal.science/hal-03044982}

Submitted on 11 Dec 2020

HAL is a multi-disciplinary open access archive for the deposit and dissemination of scientific research documents, whether they are published or not. The documents may come from teaching and research institutions in France or abroad, or from public or private research centers.
L'archive ouverte pluridisciplinaire $\mathbf{H A L}$, est destinée au dépôt et à la diffusion de documents scientifiques de niveau recherche, publiés ou non, émanant des établissements d'enseignement et de recherche français ou étrangers, des laboratoires publics ou privés. 
Online conferences - towards a new (virtual) reality

Goedele Roos ${ }^{1, *}$, Julianna Oláh ${ }^{2}$, Rebecca Ingle ${ }^{3}$, Rika Kobayashi $^{4}$, Milica Feldt $^{5}$

${ }^{1}$ Univ. Lille, CNRS, UMR 8576 - UGSF - Unité de Glycobiologie Structurale et Fonctionnelle, Lille, France

${ }^{2}$ Department of Inorganic and Analytical Chemistry, Budapest University of Technology and Economics, Hungary

${ }^{3}$ Department of Chemistry, University College London, London, WC1H OAJ, UK

${ }^{4}$ ANU Supercomputer Facility, Canberra, Australia

${ }^{5}$ Theoretische Organische Chemie, Organisch-Chemisches Institut and Center for Multiscale Theory and Computation, Westfälische Wilhelms-Universität Münster, Münster, Germany

*corresponding author: goedele.roos@univ-lille.fr

CNRS Campus Haute-Borne

Avenue de Halley 50

BP 7047859658

Villeneuve-d'Ascq

France

Keywords: Virtual conference, Virtual Winter School on Computational Chemistry, hybrid online/in-person conference 


\begin{abstract}
The recent article: Nature 579, 327-328 (2020), ending with the phrase: "You can't just suddenly make a conference be online.", has motivated us to write about the practicalities and philosophy of running online events, drawing on our extensive experience running an annual online computational chemistry conference. Our goals for this online conference series have always been: 1) Availability; 2) Community building and 3) Supporting young scientists. In this article, we highlight the motivations behind our initiative, how this has influenced the organisation of our online meeting, and discuss the benefits as well as the drawbacks of virtual meetings. Virtual conferences may not fully replace in-person meetings, but they are rapidly becoming an accepted alternative format. We discuss the hybrid online/in-person conference format as a future possibility that may offer an opportunity to reduce the environmental impact and accessibility barriers associate with in-person meetings without comprising networking and community-building opportunities.
\end{abstract}

\title{
Highlights
}

- Virtual Winter School on Computational Chemistry, https://winterschool.cc/ running annually since 2015 .

- To meet our goals: 1) Availability; 2) Community building; 3) Supporting young scientists, we make our virtual conference free for participants.

- Active participation defines the virtual meeting as a conference rather than a series of webinars.

- Drawbacks of a virtual conference originates from significantly reduced personal contacts.

- For the future, it might be desirable to work towards a hybrid online/in-person conference format. 


\section{Introduction}

The recent article 'A year without conferences' published in Nature (16 March 2020), by Giuliana Viglione [1], ending with the phrase: "You can't just suddenly make a conference be online.", motivated us to share our extensive experience with running an annual online computational chemistry conference since February 2015 (Virtual Winter School on Computational Chemistry, https://winterschool.cc/). This series demonstrates that making an online conference is possible, and that it can be done "suddenly" too. Now, almost four months after the appearance of the Nature article, it is clear that the COVID-19 pandemic has caused a huge shake-up in both the education and conferencing landscape. We are most probably looking into a new virtual future. With this contribution we want to show the benefits and possibilities, but also the problems, arising from the current paradigm shift towards a new reality, where virtual conferences start to be the new and widely used means of communication. We do not intend to take a technical approach, nor intend to write a fulllength review, but rather highlight some ideas and the philosophy behind our online initiative, discuss the benefits as well as the drawbacks of a virtual meeting and provide new perspectives for those considering organizing their own events. In the future, we foresee a combination of the virtual and in-person events as a way of maximizing the benefits of both formats for presenters and attendees.

The World Wide Web is widely in use since 1993, but as Murray-Rust P. et al. discuss, in 1996, limited bandwidth hindered sharing of even simple graphical representations of molecules and data, a key component of computational chemistry conferences [2]. Thus, real time conferences, particularly with 'real time' participant sharing, were unthinkable at that time. With the ever-increasing availability of bandwidth, sharing visual information, even for more complex renderings and animation, is now possible. Technological improvements, particularly the possibility to transmit audio with minimal latency for $\mathrm{N}$ connected devices, have meant online conferences could evolve and move closer to 'real-time' communication. Events are no longer disconnected paper submissions and asynchronous messages but instead almost fully immersive real-time experiences.

When we started the Virtual Winter School on Computational Chemistry, there were not many online conferences and if travelling were not a possibility, it could be challenging to engage with the scientific community and the latest developments. The motivation in 2015 behind our first online Winter School was to ensure that scientific lectures could be followed by every scientist, even those who could not attend traditional conferences, and with the wish to facilitate scientific communication within this wider community.

This idea of using the internet as tool for academia and information sharing was nothing new. In 1994, Steven M. Bachrach created the first website to share chemical information. He founded the Internet Journal of Chemistry to explore what could be done with the internet technology and to investigate how the internet could enhance scientific publications [3]. A more detailed overview of electronic conferences held on the World Wide Web can be found in Murray-Rust P. et al. [2]. Here, we present a comprehensive history of online meetings in the computational chemistry community.

In 1995, Steven M. Bachrach ran the first virtual computational chemistry conference [4] (ECCC, Electronic Conference on Computational Chemistry) and Henry S. Rzepa followed this up with ECTOC (Electronic Conference on Trends in Organic Chemistry. (All the contents of these conference are still available online at http://www.ch.ic.ac.uk/ectoc/). These were made to share information among (computational and theoretical) chemists using media 
alongside regular conferences. The International Electronic Conference on Synthetic Organic Chemistry (ECSOC) started in 1997 and is still ongoing. The Committee on Computers in Chemical Education of the ACS Division of Chemical Education hosted from 1993 to 2017 the online ConfChem conference [5] (http://www.ccce.divched.org/confchem_archive). In July 2008, the Virtual Conference on Chemical Education was held as part of the $20^{\text {th }}$ International Conference of Chemical Education in Mauritius; in 2011, there was a virtual conference on the Future of Women in Chemistry and in 2012, a 1-day Winter Virtual Conference. Since 2013, Ponnadurai Ramasami has organized the VCCC, the Virtual Conference on Computational Chemistry [6](link to the first edition: https://sites.uom.ac.mu/vccc/). This series ran annually till 2015. From 2016 till 2019, the Virtual Conference on Computational Science was held annually (VCCS; link to the first edition: https://sites.uom.ac.mu/vccs2016/), which was replaced in 2020 by the Virtual Conference on Chemistry and its Applications (VCCA; http://sites2019.uom.ac.mu/vcca2020/). Inspired by these examples (see also the article of Ponnadurai Ramasami published online: http://irfd.org/events/wfsids/virtual/papers/sids_pramasami.pdf), we started the Virtual Winter School on Computational Chemistry (https://winterschool.cc/) that has been running since 2015 on a yearly basis. What the annual conferences of the Virtual Winter School have shown is that it is possible to recreate many aspects of a traditional conference, and to go beyond simply broadcasting a series of lectures to a global audience, by also including events such as poster sessions and opportunities for participant interaction.

The ongoing COVID-19 pandemic has caused a sudden change in the world's situation and in the conferencing landscape. At the beginning of the pandemic, questions were raised as to how, or whether, scientific conferences would survive the corona-shock [7]. However, many scientific communities and conference organizers have rapidly adopted virtual formats and within a very short time many well-established, large conferences are now going online; examples are GPUtech (https://www.nvidia.com/en-us/gtc/), C3DIS (http://www.c3dis.com/), the first Virtual Edition of the Wannier90 school (http://psi-k.net/generalannouncements/videos-of-the-wannier90-school-virtual-edit/) and the CLEO conference (https://www.cleoconference.org/). New online initiatives are emerging as well, as for example the GitHub site of Simon Duerr (EPFL, Lausanne, Switzerland, https://duerrsimon.github.io/VCMS/index.html), the Photonics Online Meetup (POM) [8] and the Polariton Chemistry Webinars (https://www.youtube.com/watch?v=03-tBGLYGFQ).

Moving an event online is not an insurmountable challenge, particularly once the organization of an in-person conference is finished. This is because the main elements of any conference the organizers, participants and speakers - are already there. The one additional step required to go virtual is obtaining online conferencing software. There are many commercially available conferencing software packages, for example: Zoom, Goto, Newrow and Webex. Other possibilities include live streaming talks through sites such as YouTube and Vimeo. Blending across several platforms is also possible. An interesting example of a mixedplatform meeting is 'Quantum Battles in Attoscience' (https://www.quantumbattles.com/). This original in-person event incorporated for the 2020 edition social media with livestreamed talks and real-time communication platforms such as Slack. The choice of software or platform depends on many factors, including predicted audience sizes and desired level of participant interactions. Preferably, speakers and participants are trained in how to use the conferencing software before the start of the conference. As well as the main talks or lectures, even hosting virtual poster sessions is feasible (e.g. through a forum or as Twitter 
conference,

cfr.

RSC

poster

Twitter

conference

https://www.rsc.org/events/detail/43176/2020-rscposter-twitter-conference).

\section{In person meetings: current criticism and their future}

Criticism of in-person meetings often centers on the environmental impact and logistical complications of travel associated with attending in-person events. Not everyone is able to travel to attend scientific conferences, for many reasons including financial, health or family issues or teaching duties. While recent efforts by organizations such as the Royal Society of Chemistry to introduce additional financial support to facilitate travel for those with caring responsibilities, or other organizations (including the American Chemical Society) to make childcare available at conference sites, this additional support alone is still not enough to make conference attendance and travel a possibility for many people.

The carbon footprint associated with travel to academic conferences has become another widely held concern. Although international conferences are excellent environments for scientific discussion and networking, the combined environmental cost of all the travel by the participants is huge. Many case studies show that academic conferences produce a substantial amount of $\mathrm{CO}_{2}$, of which travel accounts for a significant proportion (See For example [9]). In the discussed example of Desiere S. [10], 10\% of the participants accounted for nearly 50\% of the total emissions. These $10 \%$ of participants were travelling between 2400 and 16000 $\mathrm{km}$.

In response to these criticisms of in-person events, there have been ongoing discussions on reducing the frequencies and number of meetings and about pooling conferences; see for example the recent Nature column by Hamant, O. et al [11]. Unfortunately, there is little discussion on how this can be realized while mitigating the associated professional impact of fewer conference opportunities, of which early-career scientists with precarious positions are likely to be disproportionately affected. Virtual conferences - which can be followed from home without the need to travel and of which the recordings allow people to watch them at a suitable time - offer an excellent way to address the main criticisms associated with in-person events without impairing professional opportunities. The Virtual Winter School was therefore founded as a conference on the following principles: 1) Availability: Ensure free access to lectures given by prominent computational chemists to scientists worldwide; 2) Community building: Facilitate international communication and the exchange of ideas between specialists of different fields; 3 ) Supporting young scientists.

It is highly improbable that in-person events will ever completely disappear, given the recognized benefits of in-person meetings in facilitating multi-way exchanges between many participants (see for example [12]). We think that the future of flesh-and-blood conferences lies instead in an online/in-person hybrid format.By either broadcasting talks from the meeting online, or speakers delivering their presentations virtually, it is possible to reduce the environmental burden of participants travelling the greatest distances, which even if they only account for $10 \%$ of the attendees, account for $50 \%$ of carbon emissions [10]. Participation without travel creates the opportunity to extend conference attendance to larger audiences at minimal additional cost as well as reducing the financial and time burden of travel-related expenses. As conference attendance is generally considered as beneficial for an individual's career, this will create better opportunities for e. g. people from less well-financed institutes and for primary care givers (see [13] and references herein). There are many ways how such 
hybrid events could work in practice, including allowing participants from a well-defined local geographical area to attend an in-person event while participants outside that region can follow the conference online. If each year the region changes, the travel impact can be lowered, with limited impact on the social aspects. Hybrid conferences can also be envisaged for example when a local seminar day is organized among groups of the same or neighboring universities. Recording the lectures and/or live broadcasting them would as such make the seminar beneficial for a more people living too far to travel for one day. Many combinations of in-person and virtual formats could become feasible with additional technical developments and growing expertise in running such events.

\section{Components of an online conference: audience participation and community building}

For online meetings, a wide variety of formats can be adopted, ranging from a webinar series to a full online conference. The Virtual Winter School was founded as a conference. Key to replicating the full experience of a conference and achieving our goal of community building was to invest heavily in encouraging audience participation. Encouraging and ensuring attendee participation is a well-recognized challenge for online meetings and, at the Virtual Winter School, we seek to address this in several ways. During the talks, attendees can ask questions through the chat functionality of the conferencing software and receive live answers. Through the chat, participants are able to see who else is in attendance and discuss amongst themselves. Several guided discussion sessions are scheduled throughout the conference. During these sessions, attendees can ask questions on any topic, with no restriction to the presented lectures. We motivate participants to give a 5-minute presentation on a 'single figure presentation' (that was previously submitted to the forum of the conference website) - a visual equivalent of a 3-minute thesis. Single figure presentations are a single image, highlighting the key idea of the presented work.

Informal engagement with each other and speakers using chat boxes is possible at a series of 'virtual tea breaks'. This will not fully replace in-person networking but can serve as a start.

Over the years, we have seen a continuing interest in our Virtual Winter School and returning participants (Table 1). During the course of the conference, people from a very broad geographical area including developing countries are visiting our website (Figure 1). For the 2020 edition, the geographical location of participants, as submitted in their registration forms, is available (Figure 2), confirming indeed the very broad geographical range of participants. This shows that virtual meetings indeed provide an excellent opportunity to broaden the community of attendees and are accessible for many people and therefore we consider this aspect of virtual conferences as its greatest strength. Broadening the community may require extra efforts to outreach to people from low-funded institutes by e.g. special advertising or providing the necessary support and technology. For example, the online 'The Future of Meetings' conference (15-17 September 2020, hosted and funded by CSIRO, https://events.csiro.au/) provides small accessibility grants for invited speakers and selected attendees to reimburse costs made for items as e. g. webcam, headset, internet access or other support as childcare and local accommodation. 
Table 1: Number and distribution of the registrations to the Virtual Winter School on Computational Chemistry over the past three editions.

\begin{tabular}{|l|l|l|l|}
\hline Edition & $\begin{array}{l}\text { Edition } \\
4 \\
(2018)\end{array}$ & $\begin{array}{l}\text { Edition 5 } \\
(2019)\end{array}$ & $\begin{array}{l}\text { Edition 6 } \\
(2020)\end{array}$ \\
\hline $\begin{array}{l}\text { Total number of } \\
\text { registrations }\end{array}$ & 788 & 604 & 728 \\
\hline PhD Student & 394 & 301 & 351 \\
\hline $\begin{array}{l}\text { Post-doctoral } \\
\text { Researcher }\end{array}$ & 131 & 108 & 103 \\
\hline $\begin{array}{l}\text { Senior } \\
\text { Researcher }\end{array}$ & 80 & 55 & 72 \\
\hline Other & 183 & 140 & 202 \\
\hline $\begin{array}{l}\text { Submitted } \\
\text { posters on the } \\
\text { forum }\end{array}$ & $60 *$ & 33 & 17 \\
\hline
\end{tabular}

*Poster prizes were available. 

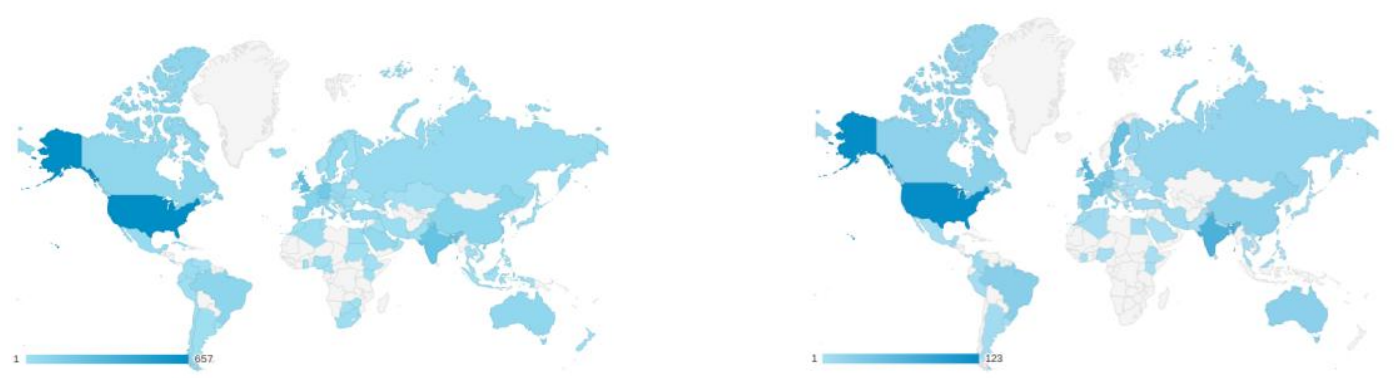

Edition 6 (2020): 89 countries

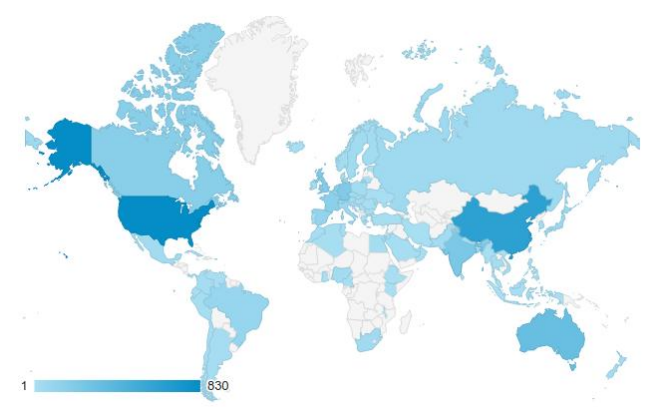

Figure 1: Geographical distribution of people visiting the winterschool.cc website (taken from Google Analytics) during the last three editions of our conference. The color code shows the total number of visits, varying from 1 (pale blue) to the maximum number of visits (dark blue). 


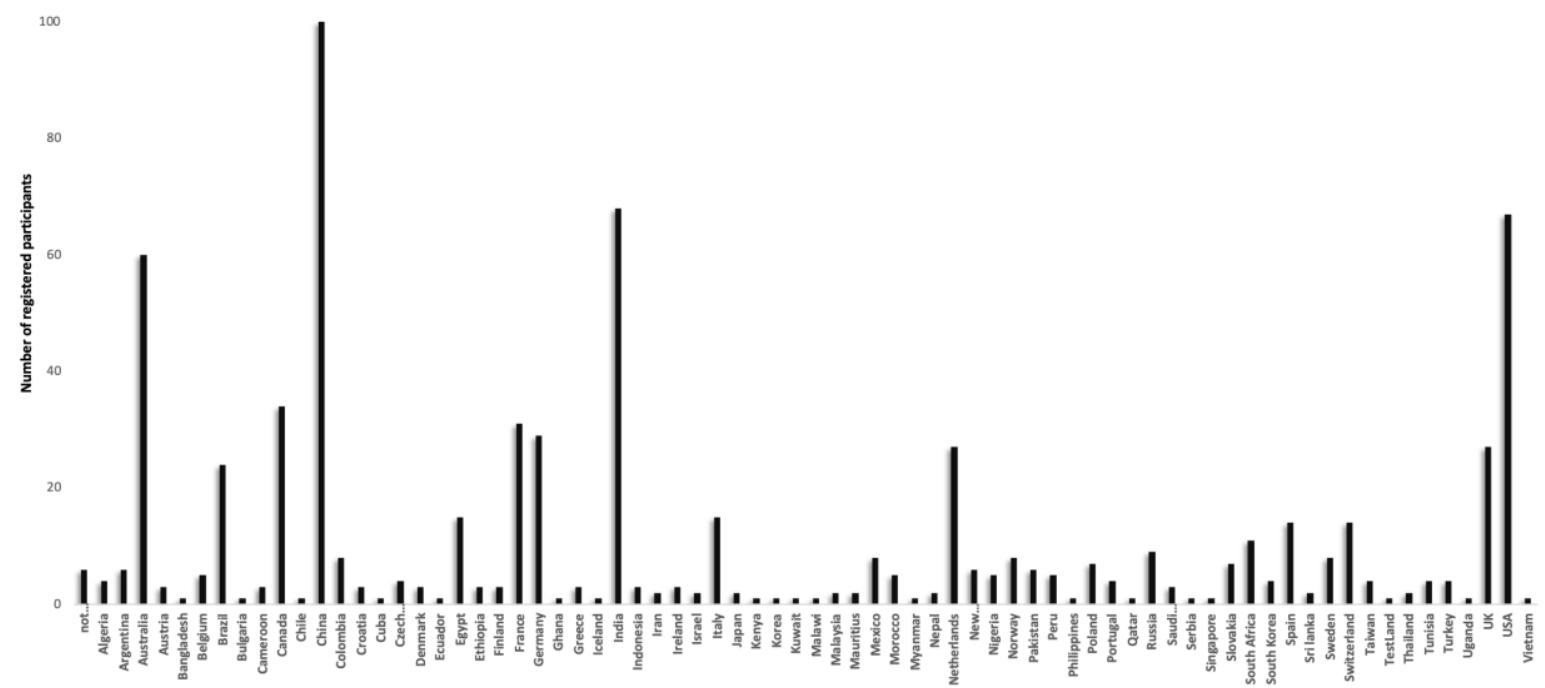

Figure 2: Number of participants registered per country for the Virtual Winter School edition 6: February 2020.

With the permission of the speaker, we record the live conference talks and have created an archive so that people can listen to them (again) at any time. Given the Virtual Winter School aims to combine pedagogical and novel research results in talks, the archive provides useful study material for graduate students and serves to attract new people in between the different editions. This adds to the creation of a community which is much broader than the traditional communities found for in-person meetings.

There are technical challenges associated with the creation and maintenance of a large archive, which Steven M. Bachrach has discussed already in 2003 [3]. There are still no collective good solutions for this despite the availability of archive.org, Web of Science, Google Scholar, Research Gate etc. We believe that setting up of a central depository for online lectures would be desirable. Until now (April 2020), our archive has 84 recordings online in the computational chemistry field. These recordings are frequently downloaded afterwards: the total number of downloads from January 2020 till the end of March 2020 thus before the world-wide COVID-19 confinement - reads 279. Lectures from early editions are still downloaded, showing a continuing interest in the recordings. Thus, many people benefit from our archive. To extend this online resource and to make recordings from other sources easily findable, we are creating - as a first step towards a central depository - a platform (https://winterschool.cc/resources) collecting links to recorded lectures to facilitate international communication on computational chemistry and the exchange of ideas between specialists of different fields. The page is created in the spirit of Wikipedia, meaning that everyone can contribute by depositing a link to a lecture you found useful or to your own recordings. In the future, it will also be possible to comment and to add information. 


\section{Costs related to the organization of a virtual conference}

Given that conference registration fees and travel can easily exceed a thousand dollars, another advantage of an online conference is the significantly reduced running costs. Compared to in-person conferences for which usually the hosting and travel expenses of the speakers need to be covered, virtual conferencing is relatively affordable.

We make the Virtual Winter School free for participants. There is a lot of ongoing discussion in a wide variety of (non)-scientific communities as to whether webinars and online conferences are worth paying for - and of course, the fear exists that a lot of bigger organizations will lose valuable revenue streams by their conferences becoming online events [7]. We believe that it is desirable to keep these events free, as financial reasons are traditionally one of the main reasons not to attend a conference. We also think it is possible to keep online conferences free as the running costs for such meetings is significantly reduced compared to a traditional conference.

It is possible to run a professional conference just for the price of the conferencing software (such as Zoom) once an official website or other information channel has been set up. Some caution should be taken when considering the cheaper price packages of virtual conferencing software tools - while they might look appealing for the budget, most conferencing software offer 'tiers' of subscription, with the cheapest ones often limiting the number of participants or the length of time a meeting can run for.

The creation of a professional website is essential but also the main hurdle to take, from a financial and/or technical point of view. While free alternatives or potentially institutional hosting might be suitable if no advanced functionality is required, the initial set up costs when professional support is required, can be significant. This is particularly challenging for 'mixed-platform' conferences that seek website integration for different aspects of the conference or communication tools. For the Virtual Winter School, hosting talk recordings also requires additional maintenance expenses but has the advantage that the content is fully within our control and, with proper archiving, can be kept secure, which may not be the case when outsourcing to third-party hosts.

Since the technical know-how for the creation of a website and the conferencing software are often the most daunting aspects of setting up an online conference, we are creating a cloneable website that can be deployed at low cost (or for free) by conference organizers. This site will be able to support large conferences with thousands of participants. A user-friendly interface will ensure that conference organizers can easily customize the content. By using open source software (as for example Joomla; the portal in which the Winter School website is built), organizers are not dependent on one hosting company or supplier. This allows flexibility, scalability, sustainability and the independence of each organizer.

\section{Drawbacks of virtual conferencing}

In the six editions of our conference (which ran in pre-COVID-19 times), we have received overwhelmingly positive feedback from participants. They mainly experience a virtual conference as beneficial. People from low-funded institutes mentioned that a virtual conference has a large impact on their careers as it is a rare (and probably the only) opportunity to have access to lectures from well-known authorities in the field. Participants 
can also discuss and present their own work and get feedback from peers from all over the world, which is for people from low-funded institutes highly validated.

While speakers also consider a virtual conference as a largely positive experience, they sometimes express that despite the questions asked after the conference, they are sometimes left with little feeling for how well the talk was received. Giving a virtual lecture is a very different experience than giving a lecture with a live audience. Sometimes speakers are reluctant to have the lecture recorded or to have it stored afterwards. It is essential to have open communication on the recording and to what happens with it afterwards. Before the start of the talk, we ask the speaker for permission to have the lecture recorded. Afterwards we send the recording first to the speaker for reviewing. In terms of storage, we offer four options to the speaker:

1. Please don't keep this recording. (it will be deleted)

2. Please make this presentation publicly accessible

3. Please make this presentation accessible for registered members

4. Please make this presentation accessible for a limited time for registered members (it will be deleted after the agreed upon period)

All these steps, together with a clear communication and the offered technical support (which was crucial in pre-COVID-19 times, when people were not so familiar yet with online events) can help to overcome initial hesitation and avoids possible problems afterwards.

A virtual conference is at the mercy of the quality of an internet connection, leading to inevitable feelings of frustration if the connection is unstable. This can be solved by providing dial-in options. Technical issues and unfamiliarity with software or platforms for speakers and attendees are another source of frustration, but can be somewhat circumvented with training events. Probably, most of these technical issues and feelings of hesitation are now overcome as people get more and more used to online events due to the COVID-19 constraints.

A major concern for online meetings is the human dimensions and psychological aspects. Scientific events are usually promoted for being an excellent source of scientific exchange, learning and networking opportunities. There is not only the scientific aspect in conferencing, but also the social aspects including cultural exchange. Travelling to an in-person meeting offers the possibility for learning about new places and cultures. Ideas pop up for virtual tourism for participants, but cannot fully replace a "real" sight-seeing experience. Participating in a virtual conference might feel much more like ordinary work, possibly just an extra activity to handle besides the daily routine. It might be much more difficult to find the time to listen to the talks as every day duties are more pressing, which - together with the very low commitment involved with registration - could also explain why usually a much lower number of registered participants actually participate in webinars and online talks. Online events do not really offer the opportunity to take a step back from the research activities, to refresh the mind or to look (literally) from a distance which most likely stimulates thinking. One of the greatest challenges with the online format is creating opportunities for informal contact and networking, as well as scientific discussions outside the appointed discussion moments. There is great interest in reproducing social inter-activity for which we are still experimenting to find the right format. With the Winter School we played with synchronous chat rooms (virtual 'tea breaks') and scheduled discussion sessions. Following the conference as a group in which the lectures can be discussed afterwards can also be a workable option to stimulate interaction. Another example of investing in the stimulation of participant interaction comes from the annual American Physical Society 
meeting (https://aps-april.onlineeventpro.freeman.com/). They provided biographies of participants and there was a matchmaking app to initiate contacts [7]. Finding creative solutions for online networking is a challenge for the whole community, but these barriers are likely to be reduced as attendees become more comfortable and experienced with in-person conferences in a virtual environment.

\section{Outlook and conclusions}

Virtual conferences have become (more and more) the new reality. This has many benefits: virtual meetings are a cheap and ecologically friendly format for knowledge transfer and scientific communication and overcomes many of the accessibility barriers surrounding traditional conferences. They are extremely powerful and bring additional value to many people and may even belittle the gap between high- and low-educated regions. But virtual meetings are also inherently impoverishing as they miss the extra dimension of the direct human-to-human interaction. The significantly reduced personal interactions between participants are considered as the main drawback of online conferences. Therefore, virtual conferences cannot (and hopefully won't) fully replace in-person meetings. Virtual meetings do not have to recreate the same types of events that could be held in person nor they should be perceived as 'lesser' as in-person events. While the ease of setting up new webinars is an excellent opportunity for bringing new voices to the scientific community, this comes with the danger of online events being created with little thought and purpose, simply because everybody else is doing so. The real challenge of any event is designing a meaningful, founded meeting giving added value for both speaker and attendee.

We see now either virtual conferences or in-person meetings. However, for the future, it might be desirable to work towards a hybrid online/in-person format to reduce pressure from environmental and availability factors without impairing social networks. Lectures from inperson conferences can be recorded or broadcasted to give the chance to a (geographically) wider audience to benefit from the lectures. This format would also be advisable to not separate the community in people attending virtual meetings and traditional conferences. Virtual conferences should not end up creating several subgroups but strive to take advantage of their inherent ability to broaden the audience. An online/in-person hybrid conference might however be a bit more complicated to set up, but with the attained knowhow of successfully held virtual conferences, not necessarily fiction and hopefully soon a fact.

\section{Extra Information}

An information webinar was held on 13 May 2020 on how to run an online conference. The recording of this session can be freely accessed here: https://winterschool.cc/how-to-run-anonline-conference.

\section{Acknowledgements}

We are very grateful to all the speakers of the virtual winter school for providing high quality lectures and to our participants for their enthusiasm and contributions. A special thanks goes to Gabor Csonka, Raom Alain Miranda Quintana, Irina Paci, Andreas Savin and Ajit Thakkar, former members of the organizing board and to all people supporting the initiative. We thank Jurgen Gaeremyn for taking care of all technical aspects of the Winter School, our coorganizer Oleg B. Gadzhiev for valuable help with registrations and forum management 
during the event, and Neepa Maitra for critical reading of the manuscript. Funding came from Royal Society of Chemistry, CNRS-Nord-Pas-de-Calais, Elsevier, CECAM-FR-MOSER. 


\section{References}

[1] G. Viglione, A year without conferences? How the coronavirus pandemic could change research, Nature. 579 (2020) 327-328. https://doi.org/10.1038/d41586-020-00786-y.

[2] P. Murray-Rust, H.S. Rzepa, B.J. Whitaker, The World-Wide Web as a chemical information tool, Chem. Soc. Rev. 26 (1997) 1-10. https://doi.org/10.1039/CS9972600001.

[3] C.K. Chamberlain, An interview with Steven Bachrach, Editor-in-Chief and Co-Founder of the Internet Journal of Chemistry, Library Collections, Acquisitions, \& Technical Services. 27 (2003) 449-458. https://doi.org/10.1080/14649055.2003.10765949.

[4] S.M. Bachrach, Electronic Conferencing on the Internet: The First Electronic Computational Chemistry Conference, J. Chem. Inf. Comput. Sci. 35 (1995) 431-441. https://doi.org/10.1021/ci00025a011.

[5] R.E. Belford, R.M. Hanson, Confchem: Web-Based Applications for Chemical Education, J. Chem. Educ. 83 (2006) 1592. https://doi.org/10.1021/ed083p1592.

[6] P. Ramasami, L. Rhyman, N. Jaufeerally, An Experience in the Digital Age, Chemistry International. 36 (2014) 8-9. https://doi.org/10.1515/ci.2014.36.4.8.

[7] G. Viglione, How scientific conferences will survive the coronavirus shock, Nature. 582 (2020) 166-167. https://doi.org/10.1038/d41586-020-01521-3.

[8] O. Reshef, I. Aharonovich, A.M. Armani, S. Gigan, R. Grange, M.A. Kats, R. Sapienza, How to organize an online conference, Nature Reviews Materials. 5 (2020) 253-256. https://doi.org/10.1038/s41578-020-0194-0.

[9] S. Jäckle, WE have to change! The carbon footprint of ECPR general conferences and ways to reduce it, European Political Science. 18 (2019) 630-650. https://doi.org/10.1057/s41304-019-00220-6.

[10] Sam Desiere, The Carbon Footprint of Academic Conferences: Evidence from the 14th EAAE Congress in Slovenia - Desiere - 2016 - EuroChoices - Wiley Online Library, (n.d.). https://onlinelibrary-wiley-com.insb.bib.cnrs.fr/doi/full/10.1111/1746-692X.12106 (accessed July 17, 2020).

[11] O. Hamant, T. Saunders, V. Viasnoff, Seven steps to make travel to scientific conferences more sustainable, Nature. 573 (2019) 451-452. https://doi.org/10.1038/d41586-019-02747-6.

[12] S. Oester, J.A. Cigliano, E.J. Hind-Ozan, E.C.M. Parsons, Why Conferences MatterAn Illustration from the International Marine Conservation Congress, Front. Mar. Sci. 4 (2017). https://doi.org/10.3389/fmars.2017.00257.

[13] L.E. Tower, M. Latimer, Cumulative Disadvantage: Effects of Early Career Childcare Issues on Faculty Research Travel, Affilia. 31 (2016) 317-330. https://doi.org/10.1177/0886109915622527. 